\title{
Early anther ablation triggers parthenocarpic fruit development in tomato
}

\author{
Mónica Medina ${ }^{1}$, Edelín Roque ${ }^{1}$, Benito Pineda ${ }^{1}$, Luis Cañas ${ }^{1}$, Manuel Rodriguez-Concepción ${ }^{2}$, José Pío Beltrán ${ }^{1}$ and \\ Concepción Gómez-Mena ${ }^{1, *}$ \\ ${ }^{1}$ Instituto de Biología Molecular y Celular de Plantas (IBMCP) CSIC-UPV, Ciudad Politécnica de la Innovación, Valencia, Spain \\ ${ }^{2}$ Centre for Research in Agricultural Genomics (CRAG) CSIC-IRTA-UAB-UB, Barcelona, Spain
}

Received 13 September 2012;

revised 19 February 2013,

accepted 7 March 2013

*Correspondence (fax +34963877859;

email cgomezm@ibmcp.upv.es)

Keywords: fruit development,

tomato, male sterility, parthenocarpy,

nutrient value.

\begin{abstract}
Summary
Fruit set and fruit development in tomato is largely affected by changes in environmental conditions, therefore autonomous fruit set independent of fertilization is a highly desirable trait in tomato. Here, we report the production and characterization of male-sterile transgenic plants that produce parthenocarpic fruits in two tomato cultivars (Micro-Tom and Moneymaker). We generated male-sterility using the cytotoxic gene barnase targeted to the anthers with the PsEND1 anther-specific promoter. The ovaries of these plants grew in the absence of fertilization producing seedless, parthenocarpic fruits. Early anther ablation is essential to trigger the developing of the transgenic ovaries into fruits, in the absence of the signals usually generated during pollination and fertilization. Ovaries are fully functional and can be manually pollinated to obtain seeds. The transgenic plants obtained in the commercial cultivar Moneymaker show that the parthenocarpic development of the fruit does not have negative consequences in fruit quality. Throughout metabolomic analyses of the tomato fruits, we have identified two elite lines which showed increased levels of several health promoting metabolites and volatile compounds. Thus, early anther ablation can be considered a useful tool to promote fruit set and to obtain seedless and good quality fruits in tomato plants. These plants are also useful parental lines to be used in hybrid breeding approaches.
\end{abstract}

\section{Introduction}

The formation of fruits in flowering plants is under both endogenous and environmental control. Hormones (mainly gibberellins and auxins) are known to have a central role in the coordinated growth of the ovary after pollination (reviewed by (de Jong et al., 2009)). The interaction between positive and negative signals controls fruit growth and part of the positive signals that promote fruit growth are thought to be produced by the developing seeds. Accordingly, fruit set is actively restricted in absence of fertilization in many species including Arabidopsis, tobacco, oilseed rape, etc. (Roque et al., 2007; Vivian-Smith et al., 2001). However, these two processes, fertilization and fruit development, can occasionally be uncoupled and the ovary can develop into a fruit without fertilization or seed formation in a process known as parthenocarpy.

In tomato, natural parthenocarpy was described several decades ago (Fos et al., 2000, 2001; George et al., 1984; Mazzucato et al., 1998; Philouze and Maisonneuve, 1978) and attracted wide interest due to its potential agronomical value. The production of seedless fruits is of great value for farmers and consumers but also for the tomato juice processing industry. Parthenocarpy has been often linked to aberrant sexual development in several species. Several tomato mutants and transgenic lines defective in stamen, anther and/or ovule development have been described to produce parthenocarpic fruit (Bishop, 1954; Mazzucato et al., 1998; Gomez et al., 1999; Ingrosso et al.,
2011). Flowers of the natural parthenocarpic apple mutant Rae Ime have no petals or stamens (Yao et al., 2001) and in the absence of manual pollination, these apetalous flowers readily bear parthenocarpic fruits. However, fruit quality of the current seedless apple mutants is poor (Yao et al., 2001).

Parthenocarpic fruit development can also be achieved by exogenous treatments with plant growth regulators even under environmental conditions adverse for fruit set and growth, but these treatments often cause malformations of the tomato fruits (Serrani et al., 2008). Genetically engineered parthenocarpic plants have been developed in tobacco, eggplant and tomato using ovule specific promoters to direct the expression of the iaaM gene to the ovules (Rotino et al., 1997, 2005). The transgenic tomato lines obtained from the processing cultivar UC 82 produce good quality fruits but seed production was not completely abolished (Rotino et al., 2005).

Genetic ablation of male tissues has been used to study male gametogenesis and as a biotechnological tool to produce male sterility in different species (De Block et al., 1997; García-Sogo et al., 2010; Mariani et al., 1990; Roberts et al., 1995; Roque et al., 2007; Rotino et al., 2005). PsEND1 (Pisum sativum ENDOTHECIUM 1) is a pea anther-specific gene that displays early expression in the anther primordium cells and in the epidermis, connective and parietal cells of anthers during development (Gómez et al., 2004). In a previous study (Roque et al., 2007), we showed that the PSEND1 promoter fused to a ribonuclease gene (barnase) from Bacillus amyloliquefaciens

Please cite this article as: Medina, M., Roque, E., Pineda, B., Cañas, L., Rodriguez-Concepción, M., Beltrán, J.P. and Gómez-Mena, C. (2013) Early anther ablation triggers parthenocarpic fruit development in tomato. Plant Biotechnol. J., doi: 10.1111/pbi.12069 
(Hartley, 1988), produced selective genetic ablation of the cell lines involved in anther architecture at early stages of development. Efficient male sterility was achieved in Arabidopsis thaliana, Brassica napus and Nicotiana tabacum transgenic plants with complete absence of fruit development (Roque et al., 2007). Here, we show that tomato plants (cv. Micro-Tom) transformed with the PSEND1::barnase construct are also male sterile. But unlike that described for other plants, these transgenic lines were found to set parthenocarpic fruits with very high efficiency. The ability of these chimeric construct to induce parthenocarpy and its effect on fruit quality parameters was tested in the commercial tomato cultivar Moneymaker.

\section{Results and discussion}

Early anther ablation causes seedless fruit development in tomato plants

Previously, we reported the use of the PSEND1::barnase construct to produce male sterile plants by early anther ablation in several species (Arabidopsis, oilseed rape, tobacco and tomato) (Roque et al., 2007). Intriguingly, tomato plants (cv. Micro-Tom) transformed with the same construct were able to set fruits in the absence of fertilization and all the fruits produced were seedless (Fig. 1). When manually pollinated with pollen from untransformed (wild-type) plants, fruits with seeds were set in all transgenic tomato lines. The average number of seed obtained using wild-type pollen $(27.8 \pm 3.1$ seeds/fruit $)$ was not significantly different to the average seed number obtained in self-pollinated wild-type flowers (33.9 \pm 3.0 seeds/ fruit).

To examine how general is the linkage between male sterility and parthenocarpy in tomato, we introduced the same construct into the tomato commercial cultivar Moneymaker. Transgenic plants were selected on the basis of the kanamycin-resistant phenotype. Ninety-six independent transgenic lines were obtained, and PCR analysed for the presence of the transgene. All lines were grown in the greenhouse and showed undeveloped stamens, male sterility and also the formation of parthenocarpic fruits (Fig. 1f,h). A subset of 10 lines exhibiting non-visual phenotypic alterations during vegetative growth were chosen for further characterization. These plants showed facultative parthenocarpy and could be manually pollinated to produce seeded fruits. The fruits obtained after manual pollination of the transgenic lines MMK TR44.2, MMK TR44.1, MMK TR67.1 and MMK TR26. 1 with wild-type pollen rendered similar number of seeds (70.3 \pm 6.4 seeds/fruit) to wild-type self-pollinated fruits $(68.1 \pm 5.2$ seeds/fruit). The six remaining lines produced a lower number of seed (28.3 \pm 5.0 seeds/fruit) that represent a $58 \%$ reduction on average compared to self-pollinated wild-type fruits. In all cases, seeds were viable and showed a normal Mendelian segregation of the selection marker. Together, these results confirmed that early anther ablation is linked to parthenocarpic fruit development in tomato and not only for the laboratory-grown miniature Micro-Tom cultivar.

It has been proposed that stamens may play a regulatory role toward the fourth floral whorl, by repressing ovary development until fertilization has taken place (Vivian-Smith et al., 2001). This repression must be exerted very early in stamen development, since manual elimination of the tomato anthers did not have any effect on promoting fruit set. Parthenocarpic fruit production and male sterility has been reported in the parthenocarpic fruit (pat) mutant of tomato although seed production is not completely abolished (Mazzucato et al., 1998). The tomato pat mutant presents aberrant anther development (Mazzucato et al., 1998). Additionally, the mutation also affects ovule development, which reduces the production of viable female gametes (Mazzucato et al., 1998). The stamenless mutant shows no stamens because these were almost completely converted into carpels. Fruits from
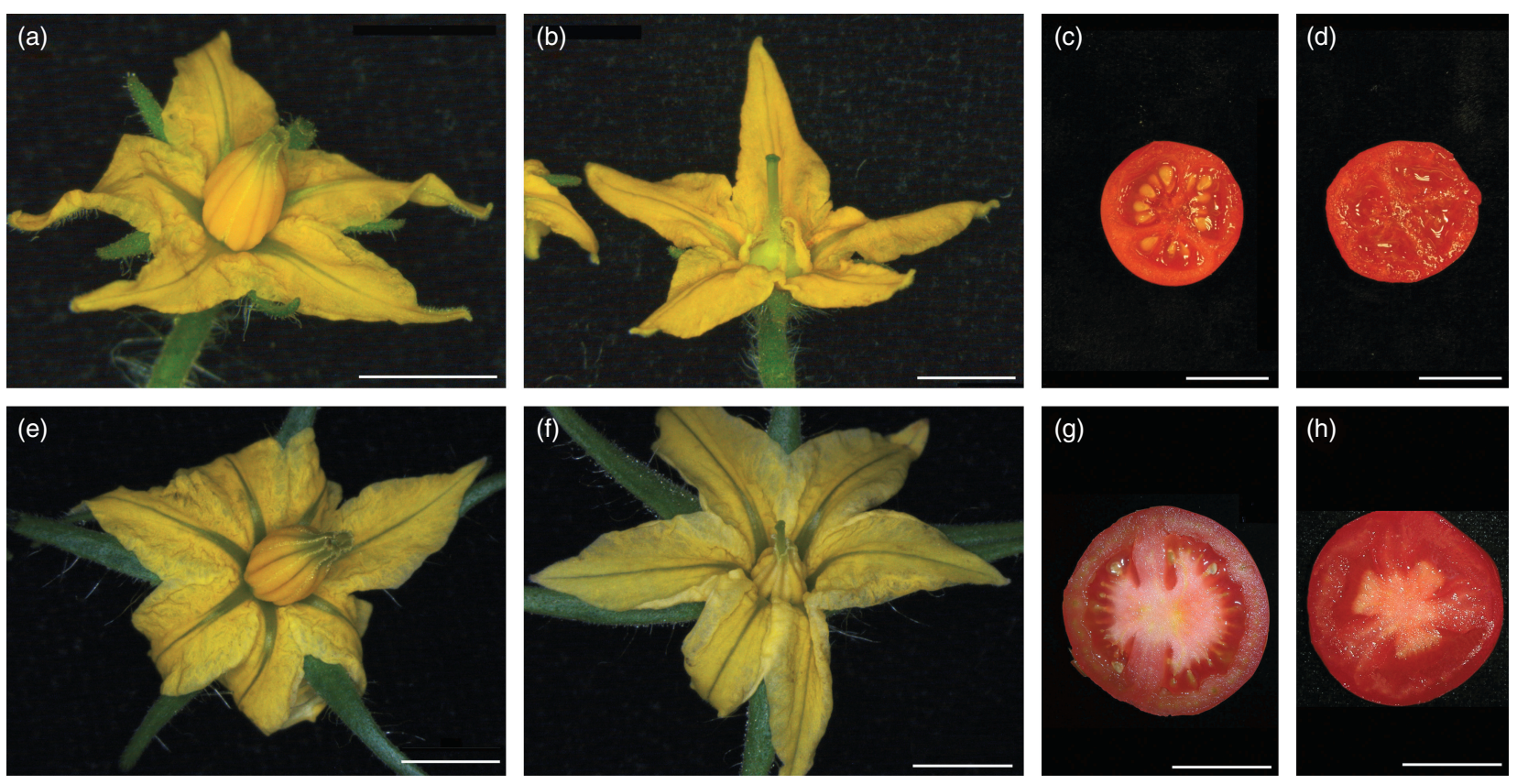

Figure 1 Male-sterile PSEND1:barnase tomato plants produce parthenocarpic fruits. Wild-type (a and e) and transgenic flowers (b and f) from Micro-Tom (upper panel) and Moneymaker (lower panel) cultivars. Transgenic flowers show aborted stamens and the pistil appears visible (b and f). Ripe fruits showing fully developed seed in the wild type ( $c$ and $g$ ) and compete absence of seeds in the transgenic lines ( $d$ and $h$ ). 
this male-sterile tomato are parthenocarpic and show roughened radial lines on the surface due to vestigial stamen development (Bishop, 1954; Gómez et al., 1999). Near complete male sterility by overexpression of a grape stilbene synthase in tomato was also associated with parthenocarpy (Ingrosso et al., 2011). A correlation between stamen disruption and parthenocarpy has additionally been reported in transgenic tomatoes where the expression of TM29, a tomato SEPALLATA homolog, was down-regulated (Ampomah-Dwamena et al., 2002). This linkage has been also reported in other species particularly in those who develop fleshy fruits. In apple, the natural mutant Rae Ime have no petals or stamens and develops seedless fruits (Yao et al., 2001). However, parthenocarpy was not always observed, associated with male-sterile genotypes (Atanassova, 2000) neither can be achieved by manual anther removal of unpollinated flowers. Early anther ablation seems to be essential to trigger the development of parthenocarpic fruits in tomato PSEND1:barnase plants. On the other hand, the transgenic lines generated in this work provide three major advantages when used as female parents: (i) the male-sterile phenotype prevents spreading of GM pollen to the environment, (ii) flowers can be easily crossed without the need of manual emasculation, and (iii) non-GM hybrid plant can be visually identified in the population resulting from the crosses because they form normal flowers. Therefore, PSEND1::barnase plants could be good parental lines to be used in breeding programs.

\section{Autonomous fruit set in PSEND1::barnase plants requires GA synthesis}

Several lines of evidence indicate that the onset of fruit development depends on the induction of gibberellin and auxin responses. In particular, the application of $\mathrm{GA}_{3}$ induced fruit set and development of emasculated ovaries in tomato (Fig. 2d). Autonomous fruit set independent of fertilization and seedless fruit production are characteristic of PSEND 1::barnase plants (Fig. 2b). To investigate whether this process depends on GAs synthesis, transgenic plants were treated with paclobutrazol (PAC), an inhibitor of P450-dependent monooxygenases that blocks GA production (Hedden and Graebe, 1985). The application of PAC completely abolished autonomous fruit set and fruit development in androesterile plants, whereas fruit development was recovered by $\mathrm{GA}_{3}$ applications to ovaries previously treated with PAC (Fig. 2C). The final size of these fruits was similar to the ones produced from untreated transgenic ovaries (Fig. 2d) but locular tissues were poorly developed (Fig. 2c) indicating that additional factors were missing for the full recovery of the internal structures of the fruit.

Changes in GA levels correlate with changes in the transcription of genes involved in GA metabolism (Lemaire-Chamley et al., 2005; Olimpieri et al., 2007; Serrani et al., 2007; Vriezen et al., 2008). Using quantitative RT-PCR, we analysed the transcription level of genes involved in GA biosynthesis (GA 20-oxidases SIGA200x1, SIGA200x2 and SIGA200x3 and GA 3-oxidases SIGA3Ox1 and S/GA3ox2) and GA deactivation (GA 2-oxidases SIGA20x1 to SIGA20x5). Ovaries from wild-type and transgenic plants were collected 6,4 and 2 days before pollination, at anthesis, and 2 days after anthesis. In both genotypes, GA20 oxidases started to rise in the ovaries before anthesis being especially dramatic in the case of SIGA200x1. This fact correlates with the moment of ovary pollination in the wild type (Fig. 3a). According to the precocious development of the transgenic ovaries, SIGA200x 1 was activated also at 6 days before anthesis and the same early activation was observed for the S/GA3ox1 transcript (Fig. 3a,b). On the other hand, the activation of SIGA3ox2 at anthesis was absent in the transgenic ovaries (Fig. 3b). In the case of the GA2-oxidases, SIGA20x1 and SIGA2ox2, both were activated after anthesis (Fig. 4) and they appear to have a relevant role in fruit development in pollinated ovaries. In the transgenic ovaries, SIGA20x2 was also activated at early stages of ovary development (Fig. 4) correlating well with the known feedback regulation mechanisms on GA biosynthesis.

Autonomous fruit set in the transgenic lines required GA synthesis and is correlated with the precocious activation of SIGA200x1 and SIGA3ox1 expression in the ovary (Fig. 3). Increased levels of GA biosynthetic genes have also been described in the pat parthenocarpic mutant (Olimpieri et al.,
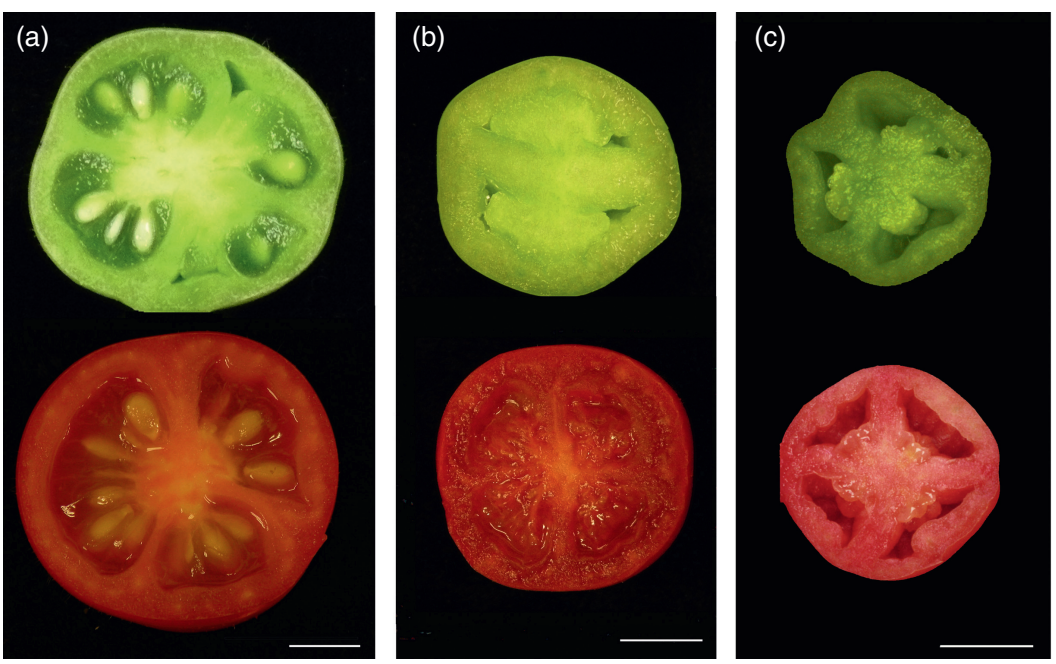

(d)

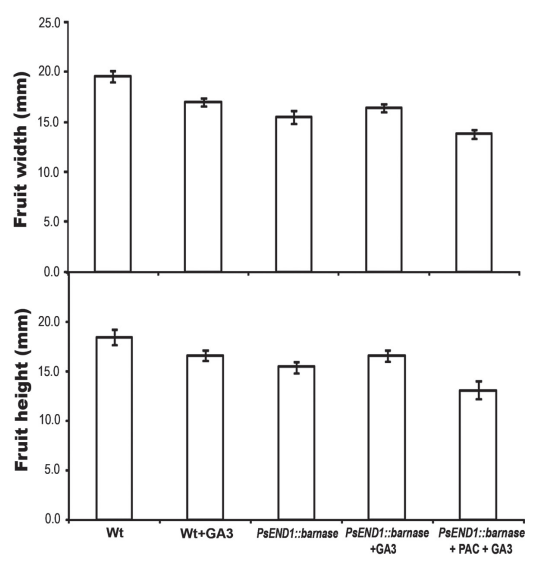

Figure 2 Parthenocarpic fruit development requires gibberellin biosynthesis. Transverse sections of representative fruits from wild type (a) collected 20 days after anthesis (top) or at maturity (bottom). (b) Parthenocarpic fruit produced by transgenic PsEND1:barnase tomato plants. (c) Paclobutrazol (PAC) treatment of the transgenic ovaries prevents the development of fruits although this effect can be restored by $\mathrm{GA}_{3}$ applications. (d) Effect of the different treatments in the size of the obtained fruits. Measurements were taken 20 days after anthesis. Scale bars in (a), (b) and (c) represent 5 mm. 

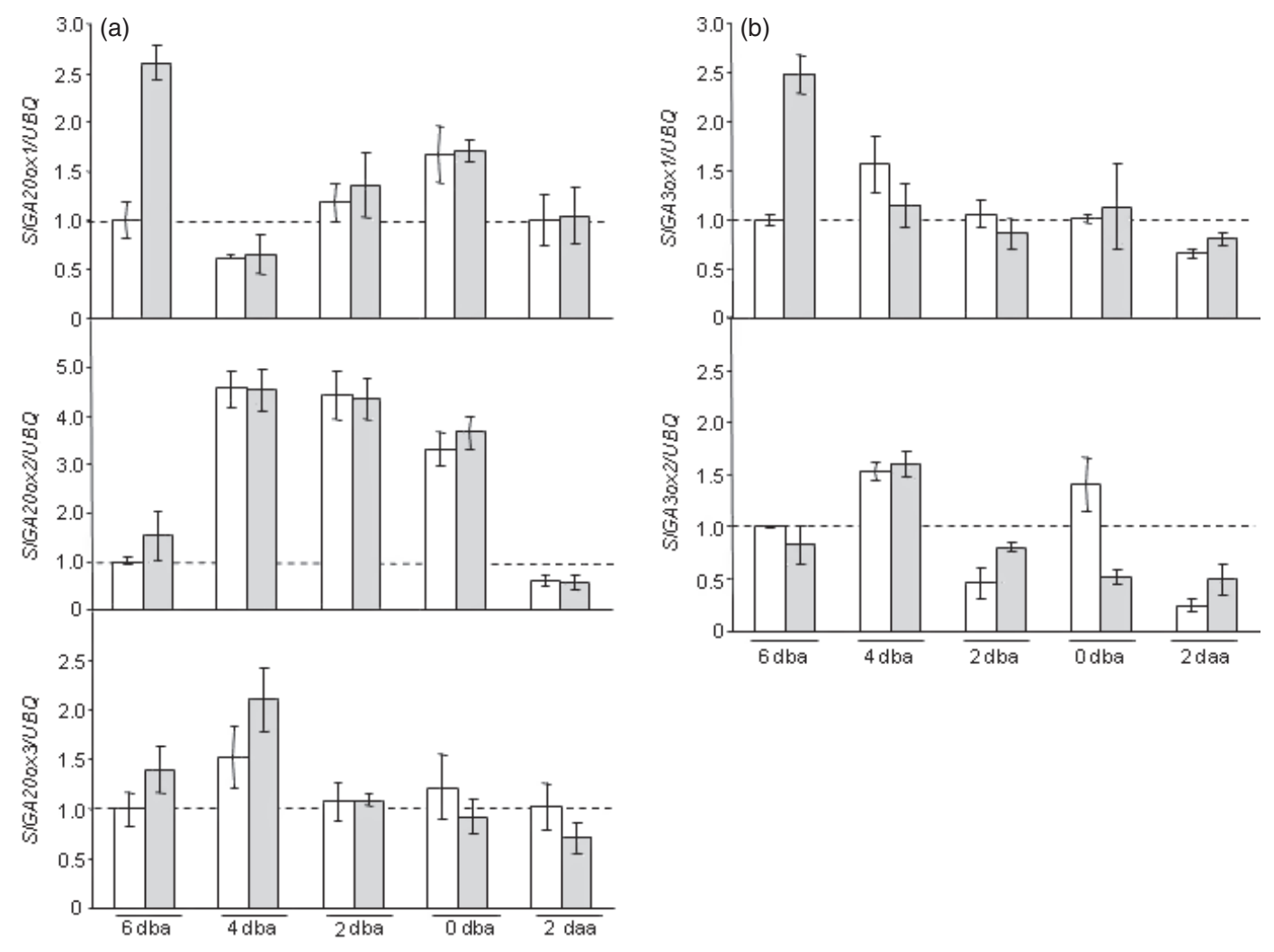

Figure 3 Changes in the expression of gibberellin biosynthesis genes, GA20-oxidases and GA3-oxidases, in the transgenic ovaries during fruit set. Wildtype (white bars) and transgenic ovaries (grey bars) where collected at 6, 4, 2 and 0 days before anthesis (dba) and 2 days after anthesis (daa). Expression of the three S/GA2O-oxidase genes (a) and the two S/GA3-oxidase genes (b) analysed by qRT-PCR. Data were normalized to the expression of UBQ and correspond to mean $( \pm \mathrm{SD})$ of three biological replicates (each with three technical replicates).

2007). Similarly, the pat-2 mutation produces the accumulation of $\mathrm{GA}_{20}$ in unpollinated tomato ovaries. Natural parthenocarpy induced by pat-2 is probably due to a higher synthesis of active GAs in the developing unpollinated ovaries (Fos et al., 2000). In naturally pollinated tomato ovaries, the expression of GA biosynthesis genes such as GA20-oxidases and GA3-oxidases was strongly up-regulated after pollination (Rebers et al., 1999; Serrani et al., 2007). In pea, the increase of GA content upon pollination is also associated with an increase in GA20 oxidase expression (van Huizen et al., 1997).

\section{Parthenocarpic fruit development does not affect fruit productivity}

We examined the performance of the transgenic phenotypes regarding fruit weight and fruit production in comparison with the untransformed wild-type genotypes. Four and ten transgenic independent lines from Micro-Tom and Moneymaker cultivars, respectively, were grown in a greenhouse together with control plants. The weight and size of the parthenocarpic fruits was analysed for all genotypes (Table 1). Transgenic plants produced fruits with lower weight in three of the four lines analysed in the Micro-Tom cultivar (Table 1). However, a significant increase in the number of fruits per plant was observed in all the plants and as a consequence plant yield, measured as the final weight of the fruits produced per plant, was not significantly affected (Table 1). Only one genotype from Micro-Tom (MT TR1d) showed an increased plant yield. The PSEND1:barnase plants produced highquality fruits that not present abnormalities except for the complete absent of seeds.
In the Moneymaker background, we observed two phenotypical classes: four lines with normal fruits and six lines that produce fruits with reduced weight (Table 1). As described for transgenic lines in the Micro-Tom background, the lines with smaller fruits also produced a higher number of fruits with respect to the untransformed controls and the final plant yield was similar to the parental lines (Table 1). Only two genotypes (MMK TR38.1 and MMK TR40.1) with the smallest fruits showed a reduction of plant yield. In all the cases, transgenic fruits did not contain any seeds. Improved fruit set in parthenocarpic tomato plants has been previously associated with a reduction in the size and weight of tomato fruits (Mazzucato et al., 1998; Rotino et al., 2005). The smaller size of the parthenocarpic fruits might be in part due to the absence of seeds. In wild-type plants, developing seeds seem to contribute to the final size of the fruits (Mapelli et al., 1978). Parthenocarpic cultivars exist in many species including tomato. However, they have not been widely used in horticulture because high yield and fruit quality is not usually combined with parthenocarpy.

\section{Transgenic parthenocarpic fruits present important changes in organoleptic quality parameters}

The parthenocarpic lines produced normal fruits without morphological external or internal defects except for the absence of seeds. A further question to analyse is whether or not the absence of developed seeds in the parthenocarpic fruits influenced other features relevant for fruit quality, including organoleptic parameters. The colour of ripe tomatoes was evaluated by colorimetry using tomato puree and the following CIELab variables 

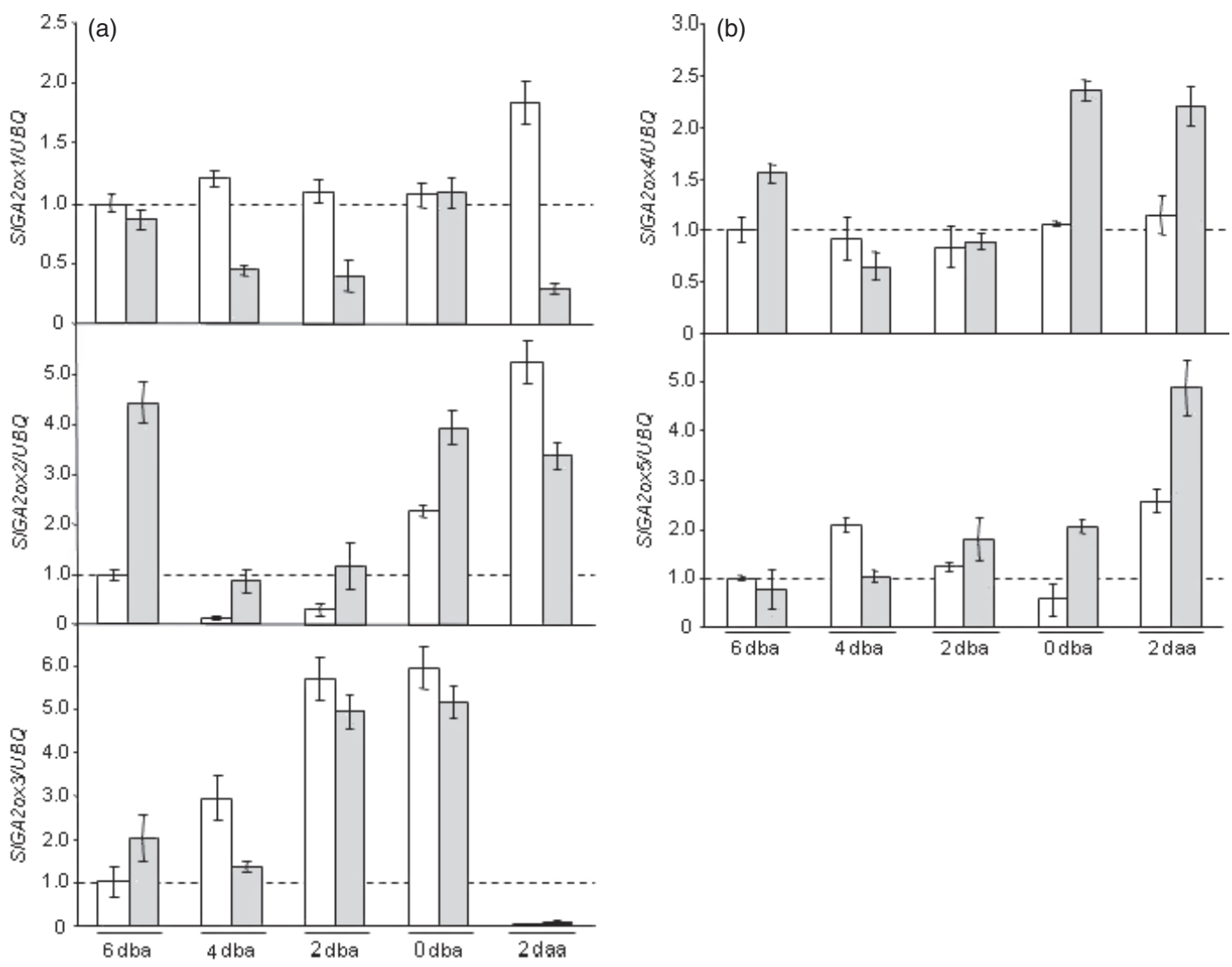

Figure 4 Changes in the expression of gibberellin inactivation genes, GA2-oxidases, in the transgenic ovaries during fruit set. Wild-type (white bars) and transgenic ovaries (grey bars) where collected at 6, 4, 2 and 0 days before anthesis (dba) and 2 days after anthesis (daa). Expression of the five SIGA2oxidase genes analysed by qRT-PCR. Data were normalized to the expression of UBQ and correspond to mean ( \pm SD) of three biological replicates (each with three technical replicates).

Table 1 Fruit weight, number of fruits per plant and plant yield for transgenic parthenocarpic lines grown under greenhouse conditions. Four independent transgenic lines obtained in the Micro-Tom cultivar and ten in the Moneymaker cultivar were analysed and compared to wild-type plants

\begin{tabular}{|c|c|c|c|c|c|}
\hline \multirow[b]{2}{*}{ Genotype } & \multicolumn{2}{|c|}{ Average size $(\mathrm{cm})$} & \multirow[b]{2}{*}{ Fruit weight (g) } & \multirow[b]{2}{*}{ Number of fruits/plant } & \multirow[b]{2}{*}{ Plant yield (g) } \\
\hline & Width & Height & & & \\
\hline MT & $19.8 \pm 0.4$ & $19.3 \pm 0.5$ & $4.7 \pm 0.1$ & $64.0 \pm 3.1$ & $305.9 \pm 6.2$ \\
\hline MT TR 15e & $19.6 \pm 1.3$ & $16.5 \pm 0.8$ & $3.9 \pm 0.5$ & $80.0 \pm 3.4$ & $315.2 \pm 4.2$ \\
\hline MT TR $1 f$ & $18.8 \pm 0.5$ & $17.3 \pm 0.4$ & $3.9 \pm 0.1$ & $75.7 \pm 6.2$ & $295.9 \pm 5.1$ \\
\hline MT TR 1d & $19.3 \pm 0.7$ & $18.9 \pm 0.7$ & $4.9 \pm 0.2$ & $73.5 \pm 3.0$ & $366.1 \pm 4.6$ \\
\hline MT TR 9L & $16.5 \pm 0.5$ & $17.3 \pm 0.6$ & $3.2 \pm 0.1$ & $91.0 \pm 2.2$ & $295.7 \pm 4.2$ \\
\hline MMK & $48.0 \pm 2.5$ & $41.2 \pm 1.8$ & $63.6 \pm 15.6$ & $29.2 \pm 1.4$ & $1857.9 \pm 39.4$ \\
\hline MMK TR38.1 & $32.9 \pm 0.3$ & $27.6 \pm 0.4$ & $27.3 \pm 4.6$ & $55.7 \pm 2.1$ & $1526.9 \pm 24.7$ \\
\hline MMK TR40.1 & $36.4 \pm 1.2$ & $29.6 \pm 0.8$ & $29.1 \pm 6.5$ & $51.5 \pm 2.1$ & $1503.5 \pm 29.1$ \\
\hline MMK TR59.1 & $41.1 \pm 2.5$ & $32.5 \pm 1.8$ & $38.6 \pm 4.7$ & $48.2 \pm 3.1$ & $1867.5 \pm 13.1$ \\
\hline MMK TR39.1 & $42.4 \pm 1.0$ & $35.0 \pm 0.5$ & $43.3 \pm 4.4$ & $42.5 \pm 2.3$ & $1847.9 \pm 27.6$ \\
\hline MMK TR48.2 & $44.3 \pm 2.5$ & $37.6 \pm 1.5$ & $44.1 \pm 3.6$ & $41.6 \pm 2.8$ & $1835.8 \pm 24.4$ \\
\hline MMK TR48.1 & $45.5 \pm 2.4$ & $36.1 \pm 1.5$ & $45.0 \pm 2.6$ & $39.5 \pm 1.2$ & $1781.0 \pm 37.5$ \\
\hline MMK TR44.2 & $43.4 \pm 2.7$ & $32.9 \pm 2.4$ & $56.9 \pm 13.6$ & $31.0 \pm 1.1$ & $1765.1 \pm 14.0$ \\
\hline MMK TR44.1 & $48.0 \pm 1.7$ & $35.3 \pm 3.4$ & $58.7 \pm 10.6$ & $31.0 \pm 2.1$ & $1822.1 \pm 11.4$ \\
\hline MMK TR67.1 & $47.2 \pm 3.2$ & $38.0 \pm 2.1$ & $63.2 \pm 6.6$ & $30.0 \pm 2.3$ & $1896.6 \pm 27.7$ \\
\hline MMK TR26.1 & $57.4 \pm 2.5$ & $42.2 \pm 1.4$ & $64.8 \pm 4.6$ & $29.6 \pm 1.2$ & $1923.3 \pm 37.4$ \\
\hline
\end{tabular}

Values represent the mean $( \pm S D)$ for ten fruits from the wild type and the transgenic lines. Statistical analyses used Student's $t$-tests $(P<0.05)$. Values in bold represent a significant increase, those in italics a significant decrease, and normal type no significant change. MT, Micro-Tom cultivar; MMK, Moneymaker cultivar. 
were obtained: $L^{*}$ (lightness), $a^{*}$ (red intensity) and $b^{*}$ (yellow intensity). The ratio $a * / b *$ was also calculated as a good indicator of ripening (Shewfelt et al., 1988). For the Micro-Tom lines, there is a homogeneous behaviour and the four lines showed fruits with higher $a^{*}$ values and lower $b^{*}$ and $L^{*}$ values compare to the wild type. These changes result in increased $a * / b *$ ratios (Table S1). Moneymaker transgenic lines do not show this common pattern. Only four lines showed increased $a * / b *$ ratios although most of the lines showed increased $a^{*}$ values (Table S1) that have been associated with lycopene synthesis (Arias et al., 2000).

To assess the possible effect of parthenocarpy in the internal quality of the fruits, we first analysed soluble solids content and titratable acidity, two parameters that are considered to be a good indicator of sensory quality. Soluble solids content ('Brix) and titratable acid was measured and the ratio ${ }^{\circ}$ Brix/acid calculated for the same lines as an indicator of maturity. Soluble solids content was higher in all four transgenic lines from the Micro-Tom cultivar (Table 2) but only two of them (MT TR15e and MT TR1f) showed a significant decrease in titratable acidity. The ${ }^{\circ}$ Brix/acid ratio increased in at least three points ranging from 12.17 to 15.80 (Table 2) in comparison with control fruits (9.08). Transgenic lines from the Moneymaker cultivar also presented higher ${ }^{\circ}$ Brix values than wild-type plants and lower titratable acidity as a common trend (Table 2 ). The ${ }^{\circ}$ Brix/acid ratio increased in all the parthenocarpic fruits with values from 8.89 to 27.40 in the independent transgenic lines. These results are in agreement with other data reported on parthenocarpic tomato fruits showing that they have higher levels of soluble solids and sugar, but lower level of acid compared with seeded fruits (Ho and Hewitt, 1986).

Volatile metabolites are major contributors to the organoleptic quality of fruits. The levels of some of these metabolites in PSEND1: barnase parthenocarpic fruits were evaluated for eight of the Moneymaker lines using five biological replicates. Fruits were harvested at ripe stage, and volatile compounds were identified and quantified using Solid Phase Microextraction (SPME) headspace extraction followed by Gas Chromatography-Mass Spectrometry (GC-MS). Principal Component Analysis (PCA) was used to express the total variation in the data set ( 53 compounds, in all 45 samples). PCA was performed to examine differences between all genotypes. The first principal component (PC1) explained $36.91 \%$ of total variability and separated samples MMKTR26.1, MMKTR59.1, MMTR38.1 and MMKTR39.1 as the most different in volatiles composition (Fig. 5a). The major contributing compounds, which explained the variations between the genotypes MMKTR59.1 and MMKTR39.1 and the wild type, were found to be increased levels of those derived from carotenoids (6-methyl-5-hepten-2-one, geranylacetone and geranial) and lipids (1-penten-3-one and (trans)-2-pentenal) and lower levels of leucine/isoleucine derived compound (3-methylbutanenitrile, 3methylbutanol and (trans)-2-methyl-2-butenal) (Fig. 5b and Table S2). For the MMKTR26.1 genotype, the differences are related to increased levels of (trans)-2-methyl-2-butenal, benzaldehyde and terpineol (Fig. 5b and Table S2). Recent results show that several volatiles including geranial make contributions to perceived sweetness independent of sugar concentrations (Tieman et al., 2012).

Changes in the level of a particular compound might have a positive or negative effect on aroma perception depending on its concentration. Lines MMKTR59.1 and MMKTR 39.1 showed increased levels of 6-methyl-5-hepten-2-one or geranylacetone, two components that contribute to add floral and fruity notes to fruits (Tandon et al., 2000). Therefore, the changes in volatiles profiles observed in these two lines might be considered as positive and would likely be beneficial to tomato flavour. Both lines were selected for a more detailed characterization at the metabolite level.

\section{Parthenocarpic fruit development results in altered metabolic profiles and high levels of phytonutrients}

The metabolite composition of ripe fruit from lines MMKTR59.1 and MMKTR 39.1, especially of those metabolites that most

Table 2 Quality variables of fruits from selected parthenocarpic genotypes compared to the untransformed control Micro-Tom (MT) or Moneymaker (MMK)

\begin{tabular}{|c|c|c|c|c|}
\hline Genotype & $\begin{array}{l}\text { Soluble solids } \\
\text { ('Brix) }\end{array}$ & $\begin{array}{l}\text { Titratable acidity } \\
\text { (\% citric acid) }\end{array}$ & $\begin{array}{l}\text { Maturity } \\
\text { index }\end{array}$ & $\begin{array}{l}\text { Flavour } \\
\text { index }\end{array}$ \\
\hline MT & $5.45 \pm 0.18$ & $0.60 \pm 0.01$ & 9.08 & 1.054 \\
\hline MT TR 15e & $8.53 \pm 0.45$ & $0.54 \pm 0.01$ & 15.80 & 1.330 \\
\hline MT TR 1f & $7.73 \pm 0.27$ & $0.57 \pm 0.01$ & 13.56 & 1.248 \\
\hline MT TR 1d & $7.18 \pm 0.20$ & $0.59 \pm 0.02$ & 12.17 & 1.198 \\
\hline MT TR 9L & $7.68 \pm 0.36$ & $0.60 \pm 0.02$ & 12.80 & 1.240 \\
\hline MMK & $4.14 \pm 0.20$ & $0.50 \pm 0.02$ & 8.28 & 0.914 \\
\hline MMK TR 38.1 & $5.00 \pm 0.01$ & $0.44 \pm 0.02$ & 11.36 & 1.008 \\
\hline MMK TR 40.1 & $4.00 \pm 0.01$ & $0.45 \pm 0.02$ & 8.89 & 0.894 \\
\hline MMK TR 59.1 & $4.70 \pm 0.21$ & $0.42 \pm 0.02$ & 11.19 & 0.980 \\
\hline MMK TR 39.1 & $6.00 \pm 0.01$ & $0.35 \pm 0.01$ & 17.14 & 1.207 \\
\hline MMK TR 48.2 & $5.00 \pm 0.01$ & $0.45 \pm 0.02$ & 11.11 & 1.006 \\
\hline MMK TR 48.1 & $6.36 \pm 0.37$ & $0.53 \pm 0.01$ & 12.00 & 1.130 \\
\hline MMK TR 44.2 & $5.00 \pm 0.45$ & $0.50 \pm 0.03$ & 10.00 & 1.000 \\
\hline MMK TR 44.1 & $5.48 \pm 0.06$ & $0.20 \pm 0.02$ & 27.40 & 1.570 \\
\hline MMK TR 67.1 & $4.88 \pm 0.16$ & $0.47 \pm 0.01$ & 10.38 & 0.989 \\
\hline MMK TR 26.1 & $5.00 \pm 0.02$ & $0.46 \pm 0.01$ & 10.87 & 1.003 \\
\hline
\end{tabular}

Statistical analyses used Student's $t$-tests $(P<0.05)$. Values in bold represent a significant increase, those in italics a significant decrease, and normal type no significant change. 

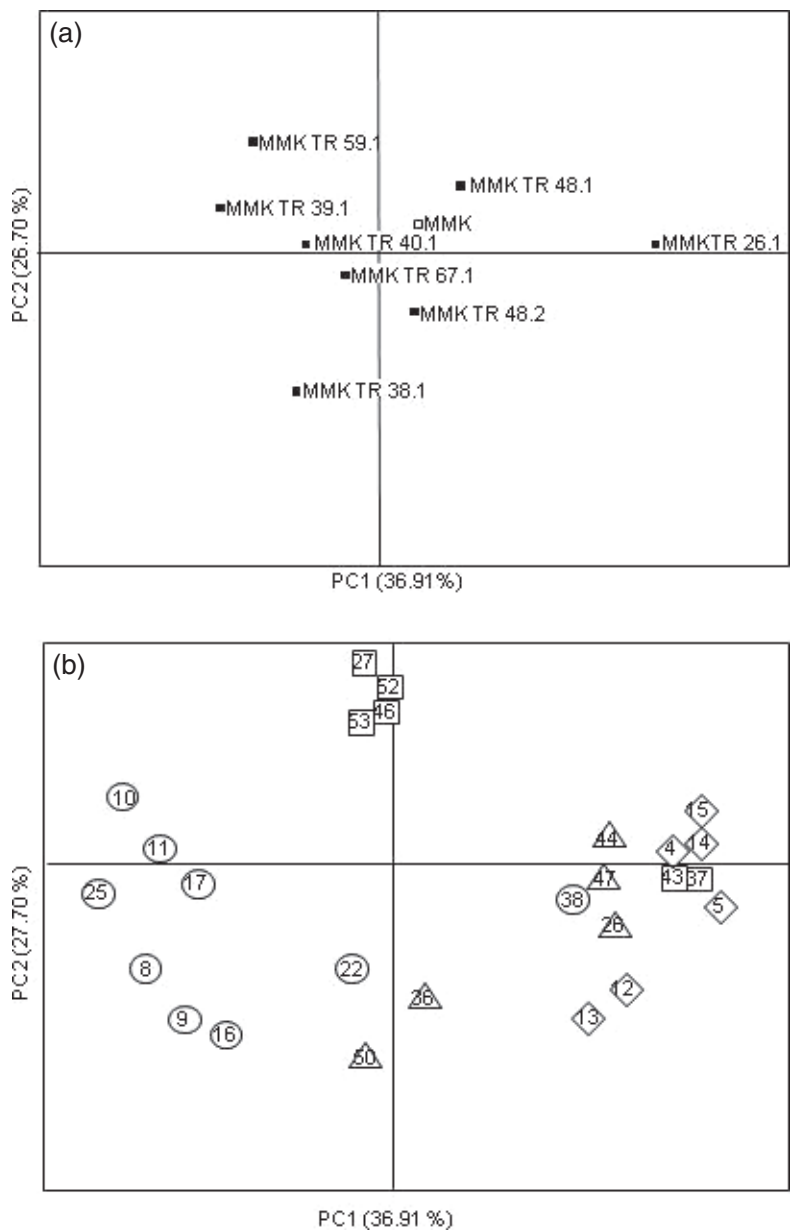

Figure 5 Volatile analysis of parthenocarpic tomatoes. (a) PCA plot generated from GC-MS data from eight independent transgenic lines and untransformed control plants (MMK). (b) PCA plot for the 26 volatile components that account for the identified variation. Numbers that identify the individual components are as in Table 2 from supplementary data. The symbols indicate the metabolic origin of the individual compounds: carotenoids (squares), phenylpropanoids (triangles), lipids (circles) are leucin (diamonds). Five biologically independent samples were analysed in triplicate for each genotype.

contribute to fruit quality and nutritional value, was analysed using five biological replicates. A total of 26 primary metabolites were quantified including most plant amino acids, organic acids, GABA, sugars and putrescine (Table 3). The data were normalized to the mean value calculated for the wild-type genotype. Line MMKTR59.1 showed higher level of all amino acids and GABA than wild type (Table 3). A similar trend was apparent for line MMKTR39.1 except for $\beta$-alanine, glycine and tyrosine that remained unchanged.

The two major organic acid constituents of fruits are citrate and malate (Mattoo et al., 1975; Tucker, 1993). Profiles of organic acids citrate and malate remained unchanged in the MMKTR59.1 line, whereas they showed modification in the line MMK39.1 where citric acid is slightly higher (1.19) and malic acid lower (0.70). Glutamic and phosphoric acid are higher in the transgenic lines and lower levels of adipic acid were detected (Table 3). The sugar that increases in both genotypes at a higher level is sucrose although MMKTR59.1 also had increased content of fructose, glucose, galactose and myo-inositol.
Table 3 Fold changes in primary metabolites relative to Moneymaker (MMK) wild-type genotype. Data were normalized to the mean value calculated for the wild-type genotype

\begin{tabular}{lll}
\hline & MMK TR 39.1 & MMK TR 59.1 \\
\hline B-Alanine & 1.04 & $\mathbf{2 . 0 4}$ \\
GABA & $\mathbf{1 . 4 7}$ & $\mathbf{1 . 7 4}$ \\
Glycine & 0.97 & $\mathbf{1 . 8 1}$ \\
Alanine & $\mathbf{1 . 4 2}$ & $\mathbf{1 . 8 3}$ \\
Asparagine & $\mathbf{2 . 0 2}$ & $\mathbf{2 . 2 3}$ \\
Aspartic acid & $\mathbf{2 . 8 4}$ & $\mathbf{2 . 6 4}$ \\
Isoleucine & $\mathbf{1 . 6 4}$ & $\mathbf{2 . 1 2}$ \\
Methionine & $\mathbf{1 . 7 7}$ & $\mathbf{2 . 9 7}$ \\
Proline & $\mathbf{1 . 6 1}$ & $\mathbf{2 . 3 5}$ \\
Tryptophan & $\mathbf{2 . 1 5}$ & $\mathbf{2 . 1 9}$ \\
Tyrosine & 1.16 & $\mathbf{2 . 0 8}$ \\
Threonine & $\mathbf{1 . 6 6}$ & $\mathbf{1 . 3 6}$ \\
Phenylalanine & $\mathbf{2 . 3 3}$ & $\mathbf{3 . 6 2}$ \\
Serine & $\mathbf{1 . 4 7}$ & $\mathbf{2 . 6 0}$ \\
Glutamic acid & $\mathbf{2 . 8 6}$ & $\mathbf{2 . 6 5}$ \\
Phosphoric acid & $\mathbf{1 . 2 3}$ & $\mathbf{1 . 5 2}$ \\
Adipic acid & 0.66 & 0.53 \\
Citric acid & $\mathbf{1 . 1 9}$ & 0.99 \\
Malic acid & 0.70 & 0.96 \\
Myo-inositol & 1.13 & $\mathbf{1 . 7 8}$ \\
Xylose & 1.08 & 1.05 \\
Galactose & 1.07 & $\mathbf{1 . 3 4}$ \\
Fructose & 1.04 & $\mathbf{1 . 1 3}$ \\
Glucose & 1.03 & $\mathbf{1 . 1 3}$ \\
Sucrose & $\mathbf{2 . 1 0}$ & $\mathbf{2 . 1 8}$ \\
Putrescine & 0.81 & $\mathbf{1 . 2 2}$ \\
\hline & & \\
\hline & &
\end{tabular}

Values are presented as the mean of five biologically independent determinations. Values in bold represent a significant increase, those in italics a significant decrease, and normal type no significant change. Statistical analyses used Student's $t$-tests $(P<0.05)$.

The red colour of ripe tomatoes is due to the presence of the carotenoid lycopene and its concentration increases with the maturity of the tomatoes. Carotenoids, and specially lycopene and beta-carotene (pro-vitamin A), play an important role in promoting human health due to their properties as biologically active signals, antioxidants and anticarcinogenic agents (Fraser et al., 2009). Tocopherols (vitamin E) are another important group of phytonutrients with antioxidant activity that share the same metabolic precursors with carotenoids (DellaPenna and Pogson, 2006). The level of both carotenoid and tocopherols in ripe tomatoes was evaluated in the parthenocarpic fruits of the transgenic lines MMKTR59.1 and MMKTR39.1 (Table 4). Both lines had significantly higher concentrations of $\beta$-carotene, most xanthophylls (oxygenated carotenoids) and tocopherols ( $\alpha$ and $\gamma$ ) compared to the wild type $(P<0.05)$. The transgenic line MMKTR39.1 also accumulated lycopene at higher levels. In this line, most carotenoids analysed increased between 2- and 2.9-fold except for neoxanthin that increased up to 4.43 -fold. Several attempts to increase carotenoid content have been made in tomato and other crop plants (Botella-Pavía and Rodríguez-Concepción, 2006; Fraser et al., 2009). However, a concomitant effect on tocopherol accumulation has only been observed in a few cases. For example, the down-regulation of the light signalling regulator 
Table 4 Fold changes in secondary metabolites relative to Moneymaker wild-type genotype. Data are normalized to the mean response calculated for the wild-type genotype

\begin{tabular}{lll}
\hline & MMK TR 39.1 & MMK TR 59.1 \\
\hline Lycopene & $\mathbf{2 . 9 0}$ & 1.12 \\
B-carotene & $\mathbf{2 . 1 1}$ & $\mathbf{1 . 4 6}$ \\
Lutein & $\mathbf{2 . 2 0}$ & $\mathbf{1 . 3 0}$ \\
Violaxanthin & $\mathbf{2 . 2 2}$ & $\mathbf{2 . 1 4}$ \\
Neoxanthin & $\mathbf{4 . 4 3}$ & $\mathbf{1 . 7 8}$ \\
Zeaxanthin & 1.13 & 1.12 \\
$\gamma$-tocopherol & $\mathbf{2 . 3 1}$ & $\mathbf{1 . 5 2}$ \\
$\alpha$-tocopherol & $\mathbf{2 . 0 7}$ & $\mathbf{1 . 2 3}$ \\
Chlorophyll a & $\mathbf{1 . 3 6}$ & 0.77 \\
Chlorophyll b & $\mathbf{1 . 3 9}$ & 0.95 \\
\hline
\end{tabular}

Values are presented as the mean of three technical independent determinations. Statistical analyses used Student's t-tests $(P<0.05)$. Values in bold represent a significant increase, those in italics a significant decrease, and normal type no significant change.

DE-ETIOLATED1 (DET1) in the fruit of the constitutive overexpression of a bacterial phytoene desaturase enzyme resulted in increased levels of both carotenoids and tocopherols in ripe fruit via an unknown mechanism (Enfissi et al., 2010; Römer et al., 2000). The elevated accumulation of both types of phytonutrients in the parthenocarpic fruit of transgenic PSEND1:barnase lines can be therefore regarded as an added value of our strategy that results in an improved nutritional quality of the fruit.

In summary, our analyses confirm early anther ablation as a highly efficient tool to induce parthenocarpic fruit development in tomato. PSEND1:barnase plants combine autonomous fruit set with the production of excellent quality fruits. Elite lines have been identified and selected for their yield and quality performance. The observed changes in the metabolic profile of the ripe fruit from such lines indicate an improved nutritional and organoleptic quality. Additionally, these male-sterile plants could be used as very convenient parental lines in hybrid breeding applications. The lines generated here can also be useful tools to investigate the molecular mechanisms responsible for the observed metabolic phenotypes but also to understand the link between impaired anther development and parthenocarpic fruit development.

\section{Experimental procedures}

\section{Plant materials and growth conditions}

Tomato plants (Solanum lycopersicum cv. Micro-Tom and cv. Moneymaker; IBMCP seed collection, Spain) were grown in pots with coconut fibre under standard glasshouse conditions at 25-30 ${ }^{\circ} \mathrm{C}$ (day) and $18-20{ }^{\circ} \mathrm{C}$ (night) and were irrigated daily with Hoagland's solution. Natural light was supplemented with Osram lamps (Powerstar HQI-BT, 400W) to get a $16 \mathrm{~h}$ light photoperiod.

\section{Genetic transformation of Solanum lycopersicum and analysis of transgenic plants}

To generate male-sterile tomato plants in the Moneymaker cultivar, we used the pBI101-PSEND1::barnase construct (Roque et al., 2007). We followed the method as described by (Ellul et al., 2003) that uses cotyledons from germinating seeds (12 days) as starting material, co-culture with A. tumefaciens (strain LBA4404) and the neomycin phosphotransferase (nptll) marker gene to carry out selection of the transformants in a kanamycin medium. Ploidy level was analysed and only the diploid lines were transferred to the greenhouse and retained for further analysis. The collection of putative transgenic tomato plants proceeding from each independent experiment was analysed by PCR to detect the presence of the barnase transgene using the primers: Ribo III (5'-ACGGACCATTAT CAGACCTTTAC-3' and Inhilll: (5'-CGCAGCCTTC-CGCTTTCGC-3') (Roque et al., 2007).

\section{Plant hormone applications}

Unless otherwise stated, three ovaries per truss, three trusses per plant and five plants were used per treatment. Application of gibberellic acid ( $\mathrm{GA}_{3}$; Duchefa, Haarlem, the Netherlands) was carried out to emasculate ovaries, the day equivalent to anthesis in $10 \mu \mathrm{L}$ of $\mathrm{GA}_{3}$ solution $\left(3 \times 10^{-4} \mathrm{M} \mathrm{GA}_{3}, 10 \%\right.$ Methanol, $1 \%$ Tween 80). Ovaries were evaluated 20 days later. Control ovaries were treated with the same volume of solvent solution. Flower emasculation was carried out 2 days before anthesis to prevent self-pollination. Paclobutrazol (PAC; Duchefa) treatments were carried out by adding PAC at $10^{-5} \mathrm{M}$ in the solution used to water the plants every 3 days.

\section{Quantitative RT-PCR}

Total RNA was extracted using the RNeasy Plant Mini Kit (Qiagen, Hilden, Germany). Genomic DNA was eliminated by treatment with 4 U rDNAsa I (RNase-free DNase set; Qiagen) for 30 min at room temperature. Three micrograms of total RNA were used to synthesize first-strand CDNA, using the SuperScript First-Strand Synthesis System for RT-PCR (Invitrogen, Carlsbad, CA). Quantitative RT-PCR was carried out using the SYBR GREEN PCR Master Mix (Applied Biosystems, Carlsbad, CA) in an ABI PRISM 7000 Sequence detection system (Applied Biosystems) following the manufacturer's recommendations.

In a single experiment, each sample was assayed in triplicate. Expression levels were calculated relative to the constitutively expressed ubiquitine gene using the $\Delta \Delta C_{t}$ method (Applied Biosystems). Primers were designed using Primer Express software from Applied Biosystems and are listed in Table S3. The amplification of ubiquitin gene was used as internal control to normalize all data (Marti et al., 2007).

\section{Morphological and chemical analyses}

The physical and chemical analyses were made in red ripe fruits harvested on the five plants of each line. Each fruit was first evaluated for physical traits: fruit weight, size, number of locules, seeds and external colour. Chemical measurements were then taken from bulk fruit: soluble solids content ( ${ }^{\circ}$ Brix) and titratable acid (TA). To minimize sample variation, pooled samples (composed from five individual fruits from at least three different plants) were considered and five independent samples were used.

The fruit samples retained for chemical determinations were each blended, filtered through a sterile mess, and the serum used for chemical analyses. Titratable acidity was determined by titrating $5 \mathrm{~mL}$ of the filtered tomato serum to $\mathrm{pH} 8.1$ with 0 . $1 \mathrm{~N} \mathrm{NaOH}$ and expressed as $\mathrm{g}$ of citric acid/100 mL, equivalent to $\%$ citric acid. Soluble solids content was expressed as ${ }^{\circ}$ Brix and was determined with a handheld refractometer (ATAGO N-14). Besides, the maturity index has been calculated as the ratio of ${ }^{\circ}$ Brix to titratable acidity, and the flavour index [(TSS/20 $\times$ titratable acidity) + titratable acidity] has also been calculated. 


\section{Volatile analysis}

For volatiles analysis, another five fruits per line (from eight genotypes) were collected and sections of the fruit were stored at $-80{ }^{\circ} \mathrm{C}$ until further use.

Fruit volatile analysis was performed essentially as described in Tikunov et al., 2005, with minor variations. Frozen tomato samples were milled in liquid nitrogen and a $1 \mathrm{~g}$ aliquot of the frozen fruit powder was used for HS-SPME-GCMS analysis. The volatiles were extracted by exposing a $65 \mu \mathrm{m}$ polydimethylsiloxanedivinylbenzene SPME fibre (Supelco, Bellefonte, PA) to the vial headspace for 20 min under continuous agitation and heating at $50{ }^{\circ} \mathrm{C}$. The fibre was manually inserted into a Clarus 500 (PerkinElmer, Wellesley, MA) injection port and volatiles were desorbed for $1 \mathrm{~min}$ at $250{ }^{\circ} \mathrm{C}$. Chromatography was performed on a ZB-5 column with helium as carrier gas, at a constant flow of

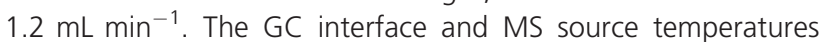
were $260^{\circ} \mathrm{C}$ and $180^{\circ} \mathrm{C}$, respectively. The oven programming conditions were $40{ }^{\circ} \mathrm{C}$ for $2 \mathrm{~min}, 5^{\circ} \mathrm{C} \mathrm{min}{ }^{-1}$ ramp until $180{ }^{\circ} \mathrm{C}$, then a $15^{\circ} \mathrm{C} \mathrm{min}^{-1}$ ramp until $250^{\circ} \mathrm{C}$, and a final hold at $250^{\circ} \mathrm{C}$ for $4 \mathrm{~min}$. The total run time, including oven cooling, was $60 \mathrm{~min}$. Mass spectra in the $35-250 \mathrm{~m} / \mathrm{z}$ range were recorded by a Clarus 500 electron impact MS (Perkin-Elmer) at a scanning speed of five scans $\mathrm{s}^{-1}$ and an ionization energy of $70 \mathrm{eV}$. The chromatography and spectral data were evaluated using TurboMass software version 5.0 (Perkin-Elmer).

\section{Primary metabolite analysis}

Metabolite analysis by GC-MS was carried out by a method modified from that described by Roessner et al., 2000;. Five fruits per line (of two genotypes) were peeled and pericarp maintained frozen at $-80{ }^{\circ} \mathrm{C}$. Tomato powder was homogeneized with liquid nitrogen and extracted in $1000 \mu \mathrm{L} \mathrm{100 \%}$ methanol and $120 \mu \mathrm{L}$ internal standard $(0.2 \mathrm{mg}$ ribitol in $1 \mathrm{~mL}$ of water). The mixture was extracted for $15 \mathrm{~min}$ at $70{ }^{\circ} \mathrm{C}$ and mixed vigorously with 1 volume of water; subsequently, the extract was centrifuged at $400 \mathrm{~g}$. Aliquots of the methanol/ water supernatant were dried in vacuo for 6-16 h. Resultant residues were redissolved in and derivatized for $2 \mathrm{~h}$ at $37{ }^{\circ} \mathrm{C}$ (in $60 \mu \mathrm{L}$ of $30 \mathrm{mg} \cdot \mathrm{mL}^{-1}$ methoxyamine hydrochloride in pyridine) followed by a 30 min treatment with $120 \mu \mathrm{L}$ of $N$-methyl-N[trimethylsilyl]trifluoroacetamide at $37{ }^{\circ} \mathrm{C}$. Twelve microliters of a retention time standard mixture $(3.7 \%[\mathrm{w} / \mathrm{v}]$ heptanoic acid, $3.7 \%[\mathrm{w} / \mathrm{v}]$ nonanoic acid, 3.7\% [w/v] undecanoic acid, 3.7\% $[\mathrm{w} / \mathrm{v}]$ tridecanoic acid, 3.7\% [w/v] pentadecanic acid, $7.4 \%[\mathrm{w} / \mathrm{v}]$ nonadeanoic acid, 7.4\% [w/v] tricosanoic acid, 22.2\% [w/v] heptacosanoic acid and 55.5\% [w/v] hentriacontanoic acid dissolved in $10 \mathrm{mg} / \mathrm{mL}$ tetrahydrofuran) was added before trimethylsilylation.

The GC-MS system used comprised a GC gas cromatograph (Agilent 6890N, Agilent Technologies Inc., Santa Clara, CA) and a Pegasus 4D GC-TOFMS mass spectrometer (LECO, St. Joseph, MI). The samples were automatically extracted and injected into the GC-MS via a Combi PAL autosampler (CTC Analytics, Zwingen, Switzerland).

GC was performed on a BPX35 column with $0.25-\mu \mathrm{m}$-film thickness. The injection temperature was set at $230^{\circ} \mathrm{C}$, the interface at $250^{\circ} \mathrm{C}$ and the ion surface adjusted to $200{ }^{\circ} \mathrm{C}$. Helium was used as the carrier gas at a flow rate of $1 \mathrm{~mL} \mathrm{~min}{ }^{-1}$. The analysis was performed under the following temperature program; $2 \mathrm{~min}$ at $85^{\circ} \mathrm{C}$ followed by a $15^{\circ} \mathrm{C} \mathrm{min}^{-1}$ oven temperature ramp to $360^{\circ} \mathrm{C}$. Mass spectra were recorded at
2 scan $\mathrm{s}^{-1}$ with a mass-to-charge ratio of 70-600 scanning range. Both chromatograms and mass spectra were evaluated using the LECO CHROMATOF (U. 3.32 optimized for Pegasus with TSD (True Signal Deconvolution) program.

\section{Secondary metabolite analysis}

Plastidial isoprenoids (chlorophylls, carotenoids and tocopherols) were extracted and separated as described previously (Roessner et al., 2000). The non-plant carotenoid canthaxanthin was used as an internal standard. Separation was carried out with an Agilent $1200 \mathrm{HPLC}$ system using a reverse-phase YMC $3 \mu \mathrm{m}$ $(4.6 \times 250 \mathrm{nn})$ C30 column. Eluted chlorophylls and carotenoids were monitored using a phosphodiode array detector, whereas tocopherols were identified using a fluorescence detector. Commercial standards were used for quantification based on peak areas.

\section{Data analysis}

Statistical analyses were performed using Microsoft Excel 7.0 (Microsoft, 2000). If two observations are described as different, this means that their difference was determined to be statistically significant $(P<0.05)$ by the performance of Student's t-tests. The transgenic genotypes were evaluated by using Student's $t$-tests at a significance threshold of 0.05 to compare statistically each trait of transgenic line with reference control. Principal component analysis was performed by means of SIMCA-P 11 software (Umetrics).

\section{Acknowledgements}

This work was supported by grants from the Spanish Ministerio de Ciencia e Innovación (MICINN, AGL2009-07617 to M.M.) and the Ramón y Cajal Program (RYC-2007-00627 to C.G.-M.). We thank Teresa Caballero, Aurora Medina, Rosa Rodríguez and José Luis Rambla for technical assistance with metabolomic analysis and Rafael Martinez for expert plant care.

The authors declare no conflict of interest.

\section{References}

Ampomah-Dwamena, C., Morris, B.A., Sutherland, P., Veit, B. and Yao, J.-L. (2002) Down-regulation of TM29, a tomato SEPALLATA Homolog, causes parthenocarpic fruit development and floral reversion. Plant Physiol., 130, 605-617.

Arias, R., Lee, T.-C., Logendra, L. and Janes, H. (2000) Correlation of Lycopene Measured by HPLC with the L*, a*, b* Color Readings of a Hydroponic Tomato and the Relationship of Maturity with Color and Lycopene Content. J. Agric. Food. Chem., 48, 1697-1702.

Atanassova, B. (2000) Functional male sterility in tomato (Lycopersicon esculentum Mill.) and its application in hybrid seed production. Acta Physiologiae Plantarum, 22, 221-225.

Bishop, C.J. (1954) A stamenless male-sterile tomato. Am. J. Bot., 41, 540-542. Botella-Pavía, P. and Rodríguez-Concepción, M. (2006) Carotenoid biotechnology in plants for nutritionally improved foods. Physiol. Plant., 126, 369-381.

De Block, M.D., Debrouwer, D. and Moens, T. (1997) The development of a nuclear male sterility system in wheat. Expression of the barnase gene under the control of tapetum specific promoters. Theor. Appl. Genet., 95, 125-131. DellaPenna, D. and Pogson, B.J. (2006) Vitamin synthesis in plants: tocopherols and carotenoids. Annu. Rev. Plant Biol., 57, 711-738.

Ellul, P.E., Garcia-Sogo, B.G.-S., Pineda, B.P., Ríos, G.R., Roig, L.R. and Moreno, V.M. (2003) The ploidy level of transgenic plants in Agrobacterium-mediated transformation of tomato cotyledons (Lycopersicon esculentum L.Mill.) is genotype and procedure dependent. Theor. Appl. Genet., 106, 231-238. 
Enfissi, E.M.A., Barneche, F., Ahmed, I., Lichtlé, C., Gerrish, C., McQuinn, R.P., Giovannoni, J.J., Lopez-Juez, E., Bowler, C., Bramley, P.M. and Fraser, P.D. (2010) Integrative transcript and metabolite analysis of nutritionally enhanced DE-ETIOLATED1 downregulated tomato fruit. Plant Cell, 22, 1190-1215.

Fos, M., Nuez, F. and García-Martínez, J.L. (2000) The gene pat-2, which induces natural parthenocarpy, alters the gibberellin content in unpollinated tomato ovaries. Plant Physiol., 122, 471-480.

Fos, M., Proaño, K., Nuez, F. and García-Martínez, J.L. (2001) Role of gibberellins in parthenocarpic fruit development induced by the genetic system pat-3/pat-4 in tomato. Physiol. Plant., 111, 545-550.

Fraser, P.D., Enfissi, E.M.A. and Bramley, P.M. (2009) Genetic engineering of carotenoid formation in tomato fruit and the potential application of systems and synthetic biology approaches. Arch. Biochem. Biophys., 483, 196-204.

García-Sogo, B., Pineda, B., Castelblanque, L., Antón, T., Medina, M., Roque, E., Torresi, C., Beltrán, J., Moreno, V. and Cañas, L. (2010) Efficient transformation of Kalanchoe blossfeldiana and production of male-sterile plants by engineered anther ablation. Plant Cell Rep., 29, 61-77.

George, W.L., Scott, J.W. and Splittstoesser, W.E. (1984) Parthenocapy in tomato. Hort. Rev., 6, 65-84.

Gómez, P., Jamilena, M., Capel, J., Zurita, S., Angosto, T. and Lozano, R. (1999) Stamenless, a tomato mutant with homeotic conversions in petals and stamens. Planta, 209, 172-179.

Gómez, M.D., Beltrán, J.-P. and Cañas, L.A. (2004) The pea END1 promoter drives anther-specific gene expression in different plant species. Planta, 219, 967-981.

Hartley, R.W. (1988) Barnase and barstar expression of its cloned inhibitor permits expression of a cloned ribonuclease. J. Mol. Biol., 202, 913-915.

Hedden, P. and Graebe, J. (1985) Inhibition of gibberellin biosynthesis by paclobutrazol in cell-free homogenates of Cucurbita maxima endosperm and Malus pumila embryos. J. Plant Growth Regul., 4, 111-122.

Ho, L.C. and Hewitt, J.D. (1986) Fruit development. In The Tomato Crop: a Scientific Basis for Improvement (Atherton, J.G. and Rudich, J., eds), pp. 201-240. Cambridge: Cambridge University Press.

van Huizen, R., Ozga, J.A. and Reinecke, D.M. (1997) Seed and hormonal regulation of gibberellin 20-oxidase expression in pea pericarp. Plant Physiol., $115,123-128$.

Ingrosso, I., Bonsegna, S., Dedomenico, S., Laddomada, B., Blando, F., Santino, A. and Giovinazzo, G. (2011) Over-expression of a grape stilbene synthase gene in tomato induces parthenocarpy and causes abnormal pollen development. Plant Physiol. Biochem., 49, 1092-1099.

de Jong, M., Mariani, C. and Vriezen, W.H. (2009) The role of auxin and gibberellin in tomato fruit set. J. Exp. Bot., 60, 1523-1532.

Lemaire-Chamley, M., Petit, J., Garcia, V., Just, D., Baldet, P., Germain, V., Fagard, M., Mouassite, M., Cheniclet, C. and Rothan, C. (2005) Changes in transcriptional profiles are associated with early fruit tissue specialization in tomato. Plant Physiol., 139, 750-769.

Mapelli, S., Frova, C., Torti, G. and Soressi, G.P. (1978) Relationship between set, development and activities of growth regulators in tomato fruits. Plant Cell Physiol., 19, 1281-1288.

Mariani, C., Beuckeleer, M.D., Truettner, J., Leemans, J. and Goldberg, R.B (1990) Induction of male sterility in plants by a chimaeric ribonuclease gene. Nature, 347, 737-741

Marti, C., Orzaez, D., Ellul, P., Moreno, V., Carbonell, J. and Granell, A. (2007) Silencing of DELLA induces facultative parthenocarpy in tomato fruits. Plant J., 52, 865-876.

Mattoo, A.K., Murata, T., Pantastico, Er.B., Chachin, K., Ogata, K. and Phan, C.T. (1975) Chemical changes during ripening and senescence. In Postharvest Physiology, Handling and Utilization of Tropical and Subtropical Fruits and Vegetables (Pantastico, E., ed.) pp. 104-127. Westport, CT: AVI Publishing.

Mazzucato, A., Taddei, A.R. and Soressi, G.P. (1998) The parthenocarpic fruit (pat) mutant of tomato (Lycopersicon esculentum Mill.) sets seedless fruits and has aberrant anther and ovule development. Development, 125, 107-114.

Olimpieri, I., Siligato, F., Caccia, R., Mariotti, L., Ceccarelli, N., Soressi, G.P. and Mazzucato, A. (2007) Tomato fruit set driven by pollination or by the parthenocarpic fruit allele are mediated by transcriptionally regulated gibberellin biosynthesis. Planta, 226, 877-888.

Philouze, J. and Maisonneuve, B. (1978) Heredity of the natural ability to set parthenocarpic fruits in the Soviet variety Severianin. Tomato Genet. Coop., 28, 12-13.
Rebers, M., Kaneta, T., Kawaide, H., Yamaguchi, S., Yang, Y.-Y., Imai, R., Sekimoto, H. and Kamiya, Y. (1999) Regulation of gibberellin biosynthesis genes during flower and early fruit development of tomato. Plant J., 17, 241-250.

Roberts, M.R., Boyes, E. and Scott, R.J. (1995) An investigation of the role of the anther tapetum during microspore development using genetic cell ablation. Sex. Plant Reprod., 8, 299-307.

Roessner, U., Wagner, C., Kopka, J., Trethewey, R.N. and Willmitzer, L. (2000) Simultaneous analysis of metabolites in potato tuber by gas chromatography-mass spectrometry. Plant J., 23, 131-142.

Römer, S., Fraser, P.D., Kiano, J.W., Shipton, C.A., Misawa, N., Schuch, W. and Bramley, P.M. (2000) Elevation of the provitamin a content of transgenic tomato plants. Nat. Biotechnol., 18, 666-669.

Roque, E., Gómez, M., Ellul, P., Wallbraun, M., Madueño, F., Beltrán, J.-P. and Cañas, L. (2007) The PsEND1 promoter: a novel tool to produce genetically engineered male-sterile plants by early anther ablation. Plant Cell Rep., 26, 313-325.

Rotino, G.L., Perri, E., Zottini, M., Sommer, H. and Spena, A. (1997) Genetic engineering of parthenocarpic plants. Nat. Biotechnol., 15, 1398-1401.

Rotino, G.L., Acciarri, N., Sabatini, E., Mennella, G., Lo Scalzo, R., Maestrelli, A., Molesini, B., Pandolfini, T., Scalzo, J., Mezzetti, B. and Spena, A. (2005) Open field trial of genetically modified parthenocarpic tomato: seedlessness and fruit quality. BMC Biotechnol., 5, 32.

Serrani, J., Fos, M., Atarés, A. and García-Martínez, J. (2007) Effect of Gibberellin and Auxin on Parthenocarpic Fruit Growth Induction in the cv Micro-Tom of Tomato. J. Plant Growth Regul., 26, 211-221.

Serrani, J.C., Ruiz-Rivero, O., Fos, M. and García-Martínez, J.L. (2008) Auxininduced fruit-set in tomato is mediated in part by gibberellins. Plant J., 56, 922-934.

Shewfelt, R.L., Thai, C.N. and Davis, J.W. (1988) Prediction of changes in color of tomatoes during ripening at different constant temperatures. J. Food Sci., 53, 1433-1437.

Tandon, K.S., Abegaz, E., Shewfelt, R.L., Baldwin, E.A. and Scott, J.W. (2000) Interrelationship of sensory descriptors and chemical composition as affected by harvest maturity and season on fresh tomato flavor. Proc. Fla. State Hort. Soc., 113, 289-294.

Tieman, D., Bliss, P., Mclntyre, L.M., Blandon-Ubeda, A., Bies, D., Odabasi, A.Z., Rodríguez, G.R., van der Knaap, E., Taylor, M.G., Goulet, C., Mageroy, M.H., Snyder, D.J., Colquhoun, T., Moskowitz, H., Clark, D.G., Sims, C., Bartoshuk, L. and Klee, H.J. (2012) The chemical interactions underlying tomato flavor preferences. Curr. Biol., 22, 1035-1039.

Tikunov, Y., Lommen, A., de Vos, C.H.R., Verhoeven, H.A., Bino, R.J., Hall, R.D and Bovy, A.G. (2005) A novel approach for nontargeted data analysis for metabolomics Large-scale profiling of tomato fruit volatiles. Plant Physiol. 139, 1125-1137.

Tucker, G.A. (1993) Introduction. In Biochemistry of Fruit Ripening (Seymour, G.B., Taylor, J.E. and Tucker, G.A., eds), pp. 3-43. London: Chapman and Hall. Vivian-Smith, A., Luo, M., Chaudhury, A. and Koltunow, A. (2001) Fruit development is actively restricted in the absence of fertilization in Arabidopsis. Development, 128, 2321-2331.

Vriezen, W.H., Feron, R., Maretto, F., Keijman, J. and Mariani, C. (2008) Changes in tomato ovary transcriptome demonstrate complex hormonal regulation of fruit set. New Phytol., 177, 60-76.

Yao, J.-L., Dong, Y.-H. and Morris, B.A.M. (2001) Parthenocarpic apple fruit production conferred by transposon insertion mutations in a MADS-box transcription factor. Proc. Natl Acad. Sci., 98, 1306-1311.

\section{Supporting information}

Additional Supporting information may be found in the online version of this article:

Table S1 Mean values ( \pm SE) of colour coordinate $\left(L^{*}, a^{*}\right.$ and $\left.b^{*}\right)$, Chroma and Hue detected in transgenic parthenocarpic fruits from two tomato cultivars.

Table S2 Volatile analysis of parthenocarpic PSEND1:barnase fruits from the Moneymaker cultivar.

Table S3 Primers used in qRT-PCR experiments in this work. 